\title{
"These Classes Have Been my Happy Place": Feasibility Study of a Self-Care Program in Native Hawaiian Custodial Grandparents
}

Asian/Pacific Island Nursing Journal

Volume 2(3): 103-109

CAuthor(s) 2017

http://digitalscholarship.unlv.edu/apin/

\author{
Loriena Yancura $^{\text {a }}$, Heather Greenwood-Junkermeier ${ }^{\mathrm{a}}$, and Christine A. Fruhauf ${ }^{\mathrm{b}}$
}

\begin{abstract}
Native Hawaiian custodial grandparents have a distinctive set of strengths and challenges that may lead them to benefit from a structured self-care program. The purpose of this paper is to describe a feasibility study with nine Native Hawaiian custodial grandparents who participated in a 6-week self-care intervention. Based on openended questions during the post-questionnaire and at the 6-month follow-up focus group, grandparent participants noted that their grandchildren needed education and clothing. Most grandparents did not endorse statements that their grandchildren had any mental or physical health conditions. Grandparents reflected that the intervention provided them with skills to help cope with raising grandchildren and helped them realize the importance of their health to providing care to their grandchildren. Based on the findings from this pilot study, the self-care approach may have benefits for Native Hawaiian custodial grandparents.
\end{abstract}

Keywords: Native Hawaiian, custodial grandparents, self-care

The U.S. Census reports that nearly 2.7 million grandparents are solely responsible for the care of their grandchildren (Ellis \& Simmons, 2014). Research studies use a variety of terms to refer to these grandparents: grandparents raising grandchildren, grandparents in skipped-generation households, and custodial grandparents. The familial contexts in which grandparents are raising their grandchildren are even more complex and multifaceted. Most of these contexts are shaped by the middle generations' challenges, such as limited economic opportunity, illness, addiction, and legal problems (Lee, 2017). Due to social isolation, stigma (Yancura, Fruhauf, \& Greenwood, 2016), financial stressors (Bailey, Haynes, \& Letiecq, 2013), and grandchildren's trauma-related mental, emotional, and behavioral problems (Smith \& Palmieri, 2007), custodial grandparents are at risk for poor mental and physical health outcomes (Choi, Sprang, \& Eslinger, 2016; Hayslip \& Kaminski, 2005; Hayslip, Fruhauf, \& Dolbin$\mathrm{MacNab}$, 2017). These stressors may also be exacerbated by other factors often correlated with custodial grandparenthood such as the rise of single-parent families, economic pressure, and cultural norms of grandparent care (Burnette, Sun, \& Sun, 2013). This article describes a feasibility study for the implementation of an evidence-based self-care intervention,
Powerful Tools for Caregivers (PTC; Boise, Congleton, \& Shannon, 2005), with a group of Native Hawaiian custodial grandparents.

\section{Background Information}

\section{The GRANDCares Project}

This study was part of the GRANDcares project, funded by the Children, Youth, and Families At-Risk program of the United States Department of Agriculture's National Institute of Food and Agriculture, which aims to increase resilience in vulnerable grandparent-headed families. It is based on the family resilience theory, which focuses on strengthening families' communication processes, organizational patterns, belief systems, and positive outlook (Walsh, 2002). This study examines feasibility of the first of the three components of the GRANDcares project, the development and implementation of a self-care

\footnotetext{
${ }^{a}$ University of Hawai 'i at Mānoa, USA

${ }^{b}$ Colorado State University - Fort Collins, USA
}

Corresponding Author:

Loriena Yancura, Ph.D.

Professor, Deptartment of Family and Consumer Sciences

University of Hawai'i at Mānoa

Email: loriena@hawaii.edu 
Table 1. Class Themes Representing Native Hawaiian Cultural Values

\begin{tabular}{|c|c|c|}
\hline PTC Class Theme & Message/Skill & Cultural Parallel \\
\hline Taking Care of YOU & $\begin{array}{l}\text { Taking care of yourself makes it possible to } \\
\text { care for others }\end{array}$ & Communal values \\
\hline $\begin{array}{l}\text { Identifying and Reducing Personal } \\
\text { Stress }\end{array}$ & $\begin{array}{l}\text { Using positive self-talk, breathing for re- } \\
\text { laxation }\end{array}$ & Focus on strengths, Phenomenology \\
\hline $\begin{array}{l}\text { Communicating Feelings, Needs, and } \\
\text { Concerns }\end{array}$ & Good communication & Harmonious communication \\
\hline $\begin{array}{l}\text { Communicating in Challenging Situa- } \\
\text { tions }\end{array}$ & Taking others' perspectives & Harmonious communication \\
\hline Learning From Our Emotions & Managing negative emotions & Focus on strengths \\
\hline Mastering Caregiving Decisions & The Family Meeting & Importance of ohana \\
\hline
\end{tabular}

program for custodial grandparents. It will be followed by the development of a parallel self-care program for grandchildren ages 9-12 of these grandparents and webinar-based trainings to educate professionals on strengths-based solutions for custodial grandparents.

The present feasibility study focused on the first component of the GRANDcares project with a cohort of Native Hawaiian grandparents on the island of Maui, one of the four project sites. This component seeks to empower grandparents' self-care skills with the PTC curriculum (Boise et al., 2005). PTC was originally designed for family caregivers of older adults but recently redesigned for parents of children with special needs (Powerful Tools for Caregivers, 2014) and adapted for custodial grandparents for the GRANDcares project.

The PTC curriculum was modeled after the Chronic Disease Self-Management program (Lorig et al., 2001) and seeks to foster the development of emotional regulation, self-efficacy, and self-care in its participants. These skills fit well with the family theory (Walsh, 2002) because resilience of the family unit is strengthened when individual members can take care of themselves. Each course during the 6week program explored one or two "tools" designed to help participants to better manage caregiving responsibilities, reduce burnout, and access community resources. Weekly themes are listed in Table 1 . The carefully scripted curriculum was co-led by leaders who were trained and certified through the National PTC Office, but had not necessarily had group facilitation experience. Past participants and community members with caregiving experience were encouraged to become PTC trainers (Boise et al., 2005).

Since 1998, PTC classes had been delivered to family caregivers for adults with chronic health conditions. However, class leaders and participants increasingly requested a version of PTC for other types of family caregivers. From 2011-2014, PTC worked with an advisory team of researchers and practitioners to adapt and pilot the curriculum with parents of children with special needs (Powerful
Tools for Caregivers, 2016). Research demonstrated that outcomes in self-efficacy, management of emotions, and self-care behaviors were similar to those of the original script. In 2014, the GRANDcares team slightly revised this adapted PTC curriculum for parents of children with special health/behavioral needs for custodial grandparents. All caregiver "tools" remained unchanged, but some wording and scenarios were adjusted to better reflect situations of custodial grandparents.

\section{Native Hawaiian Grandparents}

The Native Hawaiian population has a disproportionately high percentage of custodial grandparents. In 2012, a little over $3 \%$ of Native Hawaiian adults over age 30 reported that they had sole responsibility for their grandchildren's care, compared to $1 \%$ of adults in the general U.S. population (Ellis \& Simmons, 2014). Some of this disproportionate representation may be due to problems stemming from cultural trauma and economic inequalities faced by Native Hawaiians over the past two centuries (Browne, Mokuau, \& Braun, 2009). Other reasons may be related to interdependent family systems (McCubbin \& Marsella, 2009) and a cultural preference for multigenerational households. Over $10.6 \%$ of Native Hawaiian adults over age 30 lived in three or four generation households, compared to $3.8 \%$ of adults in the general U.S. population (Ellis \& Simmons, 2014). Thus, the experiences of Native Hawaiian custodial grandparents are influenced by a distinctive set of strengths and challenges.

Although the Native Hawaiian culture is also a minority culture in the United States, it has a unique history. Traditional Native Hawaiian society relied on communal farming and fishing among extended family units called 'ohana that included nonbiological kin (Handy \& Pukui, 1998; Hope \& Hope, 2003). This lifestyle gave rise to a value system characterized by high importance placed on 'ohana, cooperation, and interpersonal relationships as well as a profound and phenomenological relationship with the 
natural world (McCubbin \& Marsala, 2009). Of particular relevance is the enduring practice of hanai, wherein children were turned over to their grandparents, who would provide for their basic needs and teach them skills and family history (Mokuau et al., 2017). Events occurring within modern grandparents' lifetimes have also influenced factors related to custodial grandparenthood. Native Hawaiian adults between the ages of 40-70 have lived through the interpretation of their culture through the lens of a booming tourist industry, a renaissance of traditional values (e.g., language, music, ocean navigation), various political legislation aimed at both stigmatization and reparation, and rapid lifestyle changes that contributed to high rates of stress and obesity (Browne, Mokuau, \& Braun, 2009; McCubbin \& Marsella, 2009). These events contributed to preferences for informal and family-based social services (Browne et al., 2009; Yancura, 2013), particularly kinship over foster care (Yancura \& Greenwood, 2013).

\section{The Choice of PTC for Native Hawaiian Grandparents}

The use of a strengths-based, self-efficacy, approach to grandparent interventions is recommended to foster resilience in interventions targeted toward grandparents raising grandchildren (Fruhauf \& Bundy-Fazioli, 2013). This is in contrast to the common, but erroneous, assumption that grandparents raising their grandchildren need a "second chance" to make up for their deficient parenting with the grandchildren's parents (Hayslip, Herrington, Glover, \& Pollard, 2013). It is also in line with an ecological approach to grandparent interventions (Cox, 2003; Dolbin-MacNab, 2006). Many PTC materials have been translated into languages other than English (e.g., Spanish, Korean, Vietnamese) and used with minority populations. It is known that, "the PTC program has proven to be culturally appropriate for the underserved rural and ethnic communities it servesas long as cultural, language, economic, and literacy barriers are addressed" (Boise et al., 2005, p. 577). The program it was modeled on has been successfully used with Native Hawaiian older adults (Tomioka, Braun, Compton, \& Tanoue, 2011).

The choice of the PTC curriculum for the GRANDcares project was also influenced by several specific aspects of this Native Hawaiian sociohistorical context. First, PTC is not a prescriptive approach. Leaders do not teach from a position of superiority, but rather guide participants through a variety of tools and encourage them to adopt those that are most applicable and relevant. This stance of equality is one characteristic of a decolonizing approach and particularly important in light of Native Hawaiian cultural history (Braun, Browne, Ka'opua, Kim, \& Mokuau, 2014). Second, a strengths-based approach is compatible with the Native Hawaiian worldview (Kana'iau- puni, 2005). Third, the potential for PTC classes to be led by community members (peer grandparents) is in line with Braun et al.'s (2014) recommendation that those working with indigenous elders should only be implemented with Native Hawaiian consultants or collaborators. Fourth, PTC participants' identification as caregivers, an integral part of a family system, is consistent with previous research findings that intervention efforts in this population should be motivated by the desire to fulfill social and familial responsibilities (Yancura, 2010). Fifth, self-care is a particularly appropriate approach for Native Hawaiian adults who have more chronic health problems (i.e., obesity, diabetes, heart disease) than their peers of other races (Ka`opua, Braun, Browne, Mokuau, \& Park, 2011).

\section{Method}

\section{Sample and Setting}

Custodial grandparents were recruited over a period of three months with the assistance of the Maui Unit of the Liliu`okalani Trust (formerly Queen Liliu okalani Children's Center), "founded in 1909, for the benefit of orphan and destitute children with preference given to Native Hawaiian children" (Onipaa, 2017). Nine grandparents signed up to participate in the program (eight grandmothers and one grandfather, who was married to one of the grandmothers) and completed pre-test questionnaires. Of these, six completed the program and the post-test questionnaire (five grandmothers and one grandfather), and three attended a six-month follow up focus group (two grandmothers and one grandfather). The three grandparents who did not complete the post-test questionnaire cited various reasons. One grandmother completed the program, indicated limited English proficiency, and opted not to complete the post-test questionnaire. Another withdrew from the program due to time conflicts. The third withdrew because of a family medical crisis.

All participants were raising one or more children of Native Hawaiian ancestry. Six grandparents identified as Native Hawaiian; two of which also identified as Asian. The three grandparents were not Native Hawaiians, identifying as Pacific Islander, White, and Asian. All participants gave their informed consent to participate in the study and intervention, which was approved by the University of Hawai'i at Manoa Institutional Review board (CHS\# 23779).

\section{Curriculum Implementation}

The PTC curriculum for grandparents is entirely scripted, thus certified trainers are required to implement exactly as written (Boise et al., 2005). 
While this characteristic lends itself well to program fidelity and community implementation, it does not allow for cultural adaptations within the curriculum itself. However, the GRANDcares team received permission from the authors of PTC (Legacy Caregivers, www.ptc.org) to incorporate some features to make it "friendlier" to Native Hawaiian grandparents. The first of these was the previously-discussed partnership with the Liliu okalani Trust, whose employees helped recruit grandparents and provided a welcoming and familiar place to hold class sessions. The second was to include two extra meetings into the six-week PTC curriculum, for a total of eight weeks. The first session was used to complete the pre-test, as well as allow the grandparents to share their personal histories. Due to the indigenous Native Hawaiian worldview of interconnectedness of ohana (family) members and geographical place (McCubbin \& Marsella, 2009), these introductions needed more time than in Caucasian samples. Sessions two through seven were devoted to the PTC curriculum previously described. At the end of session seven, grandparents completed the post-test. Session eight took place two weeks after session seven and included a celebration and "talk story," or sharing personal details, which is an important part of social interaction in Native Hawaiian culture (Hope \& Hope, 2003). The third feature was to provide supervised activities for the grandchildren to eliminate childcare as an obstacle to grandparent attendance. It also allowed for families to participate together as a family, thus aligning with the Native Hawaiian value of 'ohana. The fourth feature was to provide a group meal for all participant meetings because sharing food represents connection in Native Hawaiian culture (these meals were generously provided by the Liliu`okalani Trust).

\section{Evaluation Process}

\section{Measurement}

Evaluation of the feasibility of the PTC program was examined through pre- and post-test questionnaires that contained items about demographic and household characteristics, service use, and grandchildren's special needs. Participants also answered open-ended questions. The pre- and posttests, asked "Is there anything else you would like us to know about the needs of the grandchildren you are caring for?" and "Is there anything else you would like us to know about yourself, your grandchild(ren), or your situation?" The follow-up focus group contained several open-ended questions, most notably, "What changes occurred in your life as a result of participating in the GRANDcares program?" and "What changes occurred in the way your family interacts and communicates as a result of changes you have made since participating in the GRANDcares program?" Information from all of these sources was used to evaluate the feasibility of the use of PTC with these grandparents.

\section{Grandparent Household Characteristics}

The majority were custodial grandparents, although one was another biological relation to the children she was raising. Their ages ranged from 5173 years, with an average age of 65 years. Most grandparents were retired and were caring for more than one grandchild, with an average of 3.25 grandchildren. Grandchildren's ages ranged from 9 months to 17 years. Some grandmothers were the only adults in the household. Household incomes were low in contrast to the Federal Poverty Threshold. There were no significant differences in characteristics between those who completed and dropped out of the program.

\section{Grandparent Needs}

All participants indicated that their income was not adequate to meet the needs of their families (two left the question blank). Few of the grandparents endorsed any of the questions indicating that their grandchildren had any special medical/behavioral issues or needs.

Some participants volunteered additional information in response to the open-ended prompts in the questionnaire. Although these responses were not solicited, they align with the literature on custodial grandparenting. Responses to the prompt about the needs of the grandchildren included "education," "adoption," and "clothing, because they grow so fast."

Two grandparents responded to the open ended prompt for anything else we should know. One grandmother wrote, "It's been hard when you have the parents involved with the decision making for the children." A quote in response to this same prompt by another grandmother suggests that her grandchildren might have experienced some adversity and illustrates her ties to them, "All I know is I will do my best to give my grandkids love \& affection. That's all I can do, and give them a better life."

\section{Program Efficacy}

Participant opinions on the curriculum were gathered during the structured focus group session. Overall, their opinions were very positive. Specific comments described specific program characteristics. For example, one grandparent stated,

One of the things we talked about in all the different classes was taking care of ourselves. If we're not healthy, we can't take care of the grandchildren. So we have to relax first, if we're stressed or excited about things, the kids are going to 
be the same way. They are a reflection of us.

Another grandparent said, I came into the program not know [sic] population feel at what to expect, but the program gave me a different outlook on life, on myself, and taking care of my grandchildren. One thing I do now, is make sure I praise my grandchild more, because she needs to hear those things from me too.

Another comment talked about learning new skills as an older adult:

It really is not easy to raise a grandbaby at our age. And you know they're growing up with all kinds of new stuff. Going to the workshop and getting little pieces here and there, you're going to need to use those tools sometimes.

These quotes illustrate that grandparents perceived that they gained knowledge in self-care, communication skills, and ability to apply what they've learned to specific situations. Grandparents' opinions of the PTC curriculum were favorable; however, they did not like the pre- and post-questionnaires because they preferred to express their opinions in person rather than through a survey.

\section{Discussion}

The primary purpose of this study was to determine if a self-care focused intervention would be well received by Native Hawaiian custodial grandparents. Although the scripted PTC curriculum was not specifically tailored to Native Hawaiian culture, it had previously been successfully used in culturally diverse populations (Boise, et al., 2005). In addition to this, its participant-centered, strengths-based, approach appears to be in line with some Native Hawaiian cultural attributes (Tomioka et al., 2011). Preliminary evidence suggests that grandparents experienced a change in outlook from their participation. They realized that managing their own emotions and response to stress would carry over to their grandchildren. These results indicate that self-care is an appropriate approach with this population and might eventually lead to stronger and healthier grandparentheaded families.

Data from the open-ended focus group suggest that this curriculum was well received by the grandparents. In particular, grandparent participants indicated that they would use the tools presented in the workshops, specifically stress reduction and communication skills, to take care of themselves.
Evidence in similar populations has demonstrated linkages between self-care trainings and mental/physical health outcomes (Kana'iaupuni, 2005; Tomioka et al., 2011), which suggests that use of this curriculum might eventually lead to positive outcomes in grandparent participants. Caution must be made in generalizing the results of this study, due to the small sample size and unique location. The overall GRANDcares project plans to offer this intervention several times over the next three years on both Maui and the Big Island (the island of Hawai i) in addition to sites in other states, in hopes of gathering evidence for the long-term efficacy of the PTC curriculum on health outcomes in a larger sample Native Hawaiian custodial grandparents, and grandparents with other cultural identities.

Findings from this study also add to existing literature on the characteristics and needs of Native Hawaiian custodial grandparents. In accordance with previous research findings that many grandparentheaded families live in poverty (Yancura, 2013; Yancura \& Greenwood, 2013), household incomes were below the federal poverty level. This study also provided data on household characteristics. The average grandparent was raising over three grandchildren. This not only suggests that they are at risk for caregiver burden by having multiple care recipients, as has been found with caregivers for older adults in collective cultures (Evans, Coon, Belyea, \& Ume, 2016). These relatively large family sizes speak to the value that the Native Hawaiian culture places on family, preferring to raise their own blood rather than send them to foster care (Mokuau et al., 2017).

Two results of this study are also notable in light of what they tell us about the use of mainstream Western research methods with this population. First, it is interesting that relatively few grandparents reported that their grandchildren had any health or behavioral issues. This is in direct contrast to other studies of grandparent-headed families (Lee, 2017; Smith \& Palmieri, 2007). This may be due to the unwillingness of the grandparents to label their grandchildren, particularly in regard to cultural norms of upright words and harmonious communication (McCubbin \& Marsella, 2009). The second issue was the grandparents' observation that they were not comfortable with the pre- and post-questionnaires. Part of this might have been due to the low educational attainment of this specific sample, but part might also be attributed to a cultural preference for oral storytelling and communication (Hope \& Hope, 2003).

\section{Suggestions for Practice}

As befits the purpose of this feasibility study, its results yield several suggestions for practice with this population. It is important to note that these suggestions may not be generalized beyond the State 
of Hawai i. The number of individuals reporting as full or part Native Hawaiian increased by $40 \%$ between the 2000 and the 2010 U.S. Census, with relatively large numbers in California, Washington, Texas, and Utah (Hixon, Hepler, \& Kim, 2012). Due to the cultural trauma experienced by this population, the most critical suggestion is to have Native Hawaiian scholars and staff on the research and intervention team (Braun et al., 2014). It is also important to work with a service providers, such as The Liliu okalani Trust, that are known and respected in the community. Small gestures, such as allowing time for "talk story," making sure that everyone has a chance to express their opinions, and bringing food to the gatherings can also help to make members of this population feel comfortable. These additions, particularly the provision of food, may pose challenges to program sustainability, but they are worth the effort. The food does not have to be elaborate, many of the meals in this program were simply chili and rice and may be donated by local organizations for program sustainability.

\section{Conclusions}

The qualitative results of this feasibility study indicate that the scripted PTC curriculum provides an appropriate way to foster self-care and communication skills in Native Hawaiian grandparents raising grandchildren. Practitioners can adopt extracurricular practices to help these grandparents feel comfortable with the PTC intervention. These practices include offering the program through a trusted community provider, allowing time for grandparent participants to develop a group identity, and providing food at the session meetings. Future research should focus on the effect of the PTC intervention on physical and mental health outcomes in this population.

\section{Declaration of Conflicting Interests}

The author(s) declared no potential conflicts of interest concerning the research, authorship, or publication of this article.

\section{Funding}

This research was supported by United State Department of Agriculture, National Institute of Food and Agriculture, Children Youth and Families at Risk. Also, a special thanks to Kathy Shannon \& Leslie Congelton of the Powerful Tools for Caregivers (PTC) program, LeeAnn Kawaa of Lili'uokalani Trust's Maui Unit and the Tutus and Papas on Maui.

\section{References}

Bailey, S. J., Haynes, D. C., \& Letiecq, B. L. (2013). "How Can You Retire When You Still Got a Kid in School?": Economics of Raising Grandchildren in Rural Areas. Marriage \& Family Review, 49, 671-693. https://doi.org/10.1080/01494929.2013. $\underline{803009}$

Boise, L., Congleton, L., \& Shannon, K. (2005). Empowering family caregivers: The powerful tools for caregiving program. Educational Gerontology, 31, 573-586. https://doi.org/10.1080/0360127059 0962523

Braun, K. L., Browne, C. V., Ka`opua, L. S., Kim, B. J., \& Mokuau, N. (2014). Research on indigenous elders: From positivistic to decolonizing methodologies. The Gerontologist, 54, 117-126. https:// doi.org/10.1093/geront/gnt067

Browne, C. V., Mokuau, N., \& Braun, K. L. (2009). Adversity and resiliency in the lives of Native Hawaiian elders. Social Work, 54, 253-261. https://doi.org/ 10.1093/sw/54.3.253

Burnette, D., Sun, J., \& Sun, F. (2013). A comparative review of grandparent care of children in the U.S. and China. Ageing International, 38, 43-57. https://doi.org/10.1007/s12126-012-9174-z

Choi, M., Sprang, G., \& Eslinger, J. G. (2016). Grandparents raising grandchildren: A synthetic review and theoretical model for interventions. Family \& Community Health, 39, 120-128. https://doi.org/ $\underline{10.1097 / \text { fch.0000000000000097 }}$

Cox, C. (2003). Designing interventions for grandparent caregivers: The need for an ecological perspective for practice. Families in Society: The Journal of Contemporary Social Services, 84, 127-134. https://doi.org/10.1606/1044-3894.76

Dolbin-MacNab, M. L. (2006). Just like raising your own? Grandmothers' perceptions of parenting a second time around. Family Relations, 55, 564-575. https://doi.org/10.1111/j.1741-3729.2006. $\underline{00426 . x}$

Ellis, R. R. \& Simmons, T. (2014). Coresident grandparents and their grandchildren (P20-576). Washington, DC: United States Census Bureau.

Evans, B. C., Coon, D. W., Belyea, M. J., \& Ume, E. (2016). Collective care multiple caregivers and multiple care recipients in Mexican American families. Journal of Transcultural Nursing, 28, 398-407. https://doi.org/10.1177/1043659616657 878

Fruhauf, C. A., \& Bundy-Fazioli, K. (2013). Grandparent caregivers' self-care practice: Moving toward a strengths-based approach. In B. Hayslip, Jr. \& G. C. Smith (Eds.), Resilient grandparent caregivers: A strengths based perspective (pp. 88-102). New York, NY: Routledge.

Handy, E. S. C., \& Pukui, M. K. (1998). The Polynesian family system in Kau' 'u, Hawai 'i. Mutual Publishing: Honolulu.

Hayslip, B., \& Kaminski, P. L. (2005). Grandparents raising their grandchildren: A review of the literature 
and suggestions for practice. The Gerontologist, 45, 262-269. https://doi.org/10.1093/geront/45. $\underline{2.262}$

Hayslip, B., Fruhauf, C. A., \& Dolbin-MacNab, M. (2017). Grandparents raising grandchildren: What have we learned over the past decade? The Gerontologist. https://doi.org/10.1093/geront/gnx106

Hayslip, B., Herrington, R. S., Glover, R. J., \& Pollard, S. E. (2013). Assessing attitudes toward grandparents raising their grandchildren. Journal of Intergenerational Relationships, 11, 356-379. https:// doi.org/10.1080/15350770.2013.839321

Hixson, L., Hepler, B. B., \& Kim, M. O. (2012). The Native Hawaiian and other Pacific Islander population: 2010 (C2010BR-12). Washington, DC: US Census Bureau.

Hope, B., \& Hope, J. (2003). Native Hawaiian health in Hawaii: Historical highlights. Californian Journal of Health Promotion, 1, 1-9.

Ka'opua, L. S., Braun, K. L., Browne, C. V., Mokuau, N., \& Park, C-B. (2011). Why are native Hawaiians underrepresented in Hawai' $i$ 's older adult population? Exploring social and behavioral factors of longevity. Journal of Aging Research, 2011, 1-8. https://doi.org/10.4061/2011/701232

Kana'iaupuni, S. M. (2005). Ka'akālai Kū Kanaka: A call for strengths-based approaches from a Native Hawaiian perspective. Educational Researcher, 34(5), 32-38. https://doi.org/10.3102/0013189x $\underline{034005032}$

Lee, Y. (2017). A model for school professionals working with grandparent-headed families. Social Work, 62, 122-129. https://doi.org/10.1093/sw/swx007

Lorig, K. R., Sobel, D. S., Ritter, P. L., Laurent, D., \& Hobbs, M. (2001). Effect of a self-management program on patients with chronic disease. Effective Clinical Practice, 4, 256-262.

McCubbin, L., \& Marsella, A. J. (2009). Native Hawaiian culture and behavior: The cultural, historical, and situational context of knowing and being. Cultural Diversity and Ethnic Minority Psychology, 15, 374-387.

Mokuau, N., Browne, C. V., Ka`opua, L. S., Higuchi, P., Sweet, K. M., \& Braun, K. L. (2017). Native Hawaiian grandparents: Exploring Benefits and challenges in the caregiving experience. Journal of Indigenous Social Development, 4, 1-19.

Onipaa. (2017). Liliu`okalani trust. Retrieved from http://onipaa.org/pages/the-trust

Powerful Tools for Caregivers. (2014). Powerful tools for caregivers. Retrieved from https://www.powerful toolsforcaregivers.org/

Smith, G. C., \& Palmieri, P. A. (2007). Risk of psychological difficulties among children raised by custodial grandparents. Psychiatric Services, 58, 13031310. https://doi.org/10.1176/appi.ps.58.10.1303

Tomioka, M., Braun, K. L., Compton, M., \& Tanoue, L. (2011). Adapting Stanford's chronic disease selfmanagement program to Hawaii's multicultural population. The Gerontologist, 52, 121-132. https://doi.org/10.1093/geront/gnr054

Walsh, F. (2002). A family resilience framework: Innovative practice applications. Family Relations, 51, 130-137. https://doi.org/10.1111/j.1741-3729. 2002.00130.x

Yancura, L. (2010). Creating culturally sensitive brochures for grandparents raising grandchildren in $\mathrm{Ha}-$ wai' i. Health Promotion Practice, 11, 400-407.

Yancura, L. A. (2013). Service use and unmet service needs in grandparents raising grandchildren. Journal of Gerontological Social Work, 56, 473486. $\quad$ https://doi.org/10.1080/01634372.2013. $\underline{804471}$

Yancura, L. A., \& Greenwood, H., (2013). Raising grandchildren as an expression of Native Hawaiian cultural values (pp. 105-120). In B. Hayslip \& G. Smith (Eds.), Resilient grandparent caregivers: A strengths-based perspective. New York: Routledge.

Yancura, L. A., Fruhauf, C., \& Greenwood, H. (2016). Recognizing microaggressions: A tool to foster well-being in grandfamilies. Grandfamilies: The Contemporary Journal of Research, Practice, and Policy, 3(1). Retrieved from http:// scholarworks.wmich.edu/grandfamilies/vol3/iss1/6

Yancura, L.A. (2012). Justifications for Caregiving in White, Asian American, and Native Hawaiian Grandparents Raising Grandchildren. Journals of Gerontology Series B: Psychological Sciences and Social Sciences, 68, 139-144. https:// doi.org/10.1093/geronb/gbs098 